\title{
Relationship between location and size of myocardial infarction and their reciprocal influences on post-infarction left ventricular remodeling
}

\author{
Pier Giorgio Masci ${ }^{1 *}$, Javier Ganame ${ }^{2}$, Marco Francone ${ }^{3}$, Walter Desmet ${ }^{2}$, Valentina Lorenzoni ${ }^{4}$, Ilaria lacucci ${ }^{3}$, \\ Andrea Barison ${ }^{5}$, lacopo Carbone ${ }^{3}$, Luciano Agati ${ }^{3}$, Massimo Lombardi ${ }^{1}$, Stefan Janssens ${ }^{2}$, Jan Bogaert ${ }^{2}$
}

From 2011 SCMR/Euro CMR Joint Scientific Sessions

Nice, France. 3-6 February 2011

\section{Background}

Patients with anterior MI experience worse LV remodeling and dysfunction than non-anterior MI patients. It remains unclear whether this difference is due to larger MI size or whether infarct location plays a role beyond MI size.

\section{Study aim}

To assess the relationship between myocardial infarction (MI) location and size and their reciprocal influences on post-infarction left ventricular (LV) remodeling.

\section{Methods}

A cohort of 260 reperfused ST-segment elevation MI patients was studied with cardiovascular magnetic resonance (CMR) at 1-week (baseline) and 4-month (followup). Area at risk (AAR) and MI size were quantified by T2-weighted and late gadolinium enhancement imaging, respectively. Adverse LV remodeling was defined as increase in LV end-systolic volume $\geq 15 \%$ at follow-up.

\section{Results}

One-hundred twenty-seven(49\%) patients had anterior MI and 133(51\%) patients had non-anterior MI. Although the degree of myocardial salvage was similar between groups $(\mathrm{p}=0.74)$, anterior MI patients had larger AAR and MI size than non-anterior MI patients yielding worse regional and global LV function at baseline and follow-up. At univariable analysis, anterior MI was associated with increased risk of adverse LV remodeling $(\mathrm{p}=0.017)$ and lower LV ejection-fraction at follow-up ( $\mathrm{p}=0.001$ ), but not when accounted for baseline MI size. Accordingly, at multivariable analysis baseline MI size but not its location was an independent predictor of adverse LV remodeling $(\mathrm{OR}=1.061, \mathrm{p}<0.001)$ and ejection-fraction at follow-up (Beta-coefficient $=-0.255$, $\mathrm{p}<0.001)$.

\section{Conclusions}

Anterior MI patients experience more pronounced post-infarction LV remodeling and dysfunction than non-anterior MI patients due to greater magnitude of irreversible ischemic LV damage without any independent contribution of MI location.

\section{Author details}

${ }^{1}$ Fondazione Toscana/CNR "G. Monasterio", Pisa, Italy. ' Gasthuisberg

University Hospital, Leuven, Belgium. '²a Sapienza University, Rome, Italy. ${ }^{4}$ Institute of Clinical Physiology/CNR, Pisa, Italy. ${ }^{5}$ Scuola Superiore S.Anna, Pisa, Italy.

Published: 2 February 2011

doi:10.1186/1532-429X-13-S1-P84

Cite this article as: Masci et al:: Relationship between location and size of myocardial infarction and their reciprocal influences on post-

infarction left ventricular remodeling. Journal of Cardiovascular Magnetic Resonance 2011 13(Suppl 1):P84.

"Fondazione Toscana/CNR "G. Monasterio", Pisa, Italy

Full list of author information is available at the end of the article

(c) 2011 Masci et al; licensee BioMed Central Ltd. This is an open access article distributed under the terms of the Creative Commons 